\title{
«Es ist schwierig, ein guter Doktor zu sein»
}

\section{Daniel Lüthi}

Freier Journalist und Fotograf, Medientrainer, Bern

Erst gerade ist sie zurückgekehrt von den Azoren. Ferien mit ihrem Partner - Ruhe vor dem Sturm. Die Inselgruppe draussen im Atlantik ist für die Menschen hinten im Waldemmental symbolisch: Gabriela Rohrer ist für sie so etwas wie das berühmte Azorenhoch. Endlich eine neue Hoffnung für die medizinische Grundversorgung in dieser Gegend, endlich eine Nachfolge für den langjährigen Landarzt. «Ja, der Druck ist gross, so eine Art Messias zu sein, der das Wohl ins Tal bringt», kommentiert Gabriela Rohrer in der ihr eigenen, erfrischenden Offenheit.

\section{Generationenwechsel}

Ein paar Monate lang arbeiteten die beiden Generationen Hausärzte noch zusammen, Ende März ging Paul Affentranger mit 71 Jahren und nach rund 40 Jahren Tätigkeit hier in Pension. Dann wurden die Praxisräume geputzt und neu eingerichtet, und die junge Frau aus dem Unterland übernahm. "Ein Schnitt ist nötig, wenn etwas Neues entstehen soll», sagt sie, «aber ein solcher Wechsel ist für beide Seiten nicht leicht.»

Welche Elemente sollen denn den Neubeginn markieren? «Wir führen die elektronischen Patientenakten

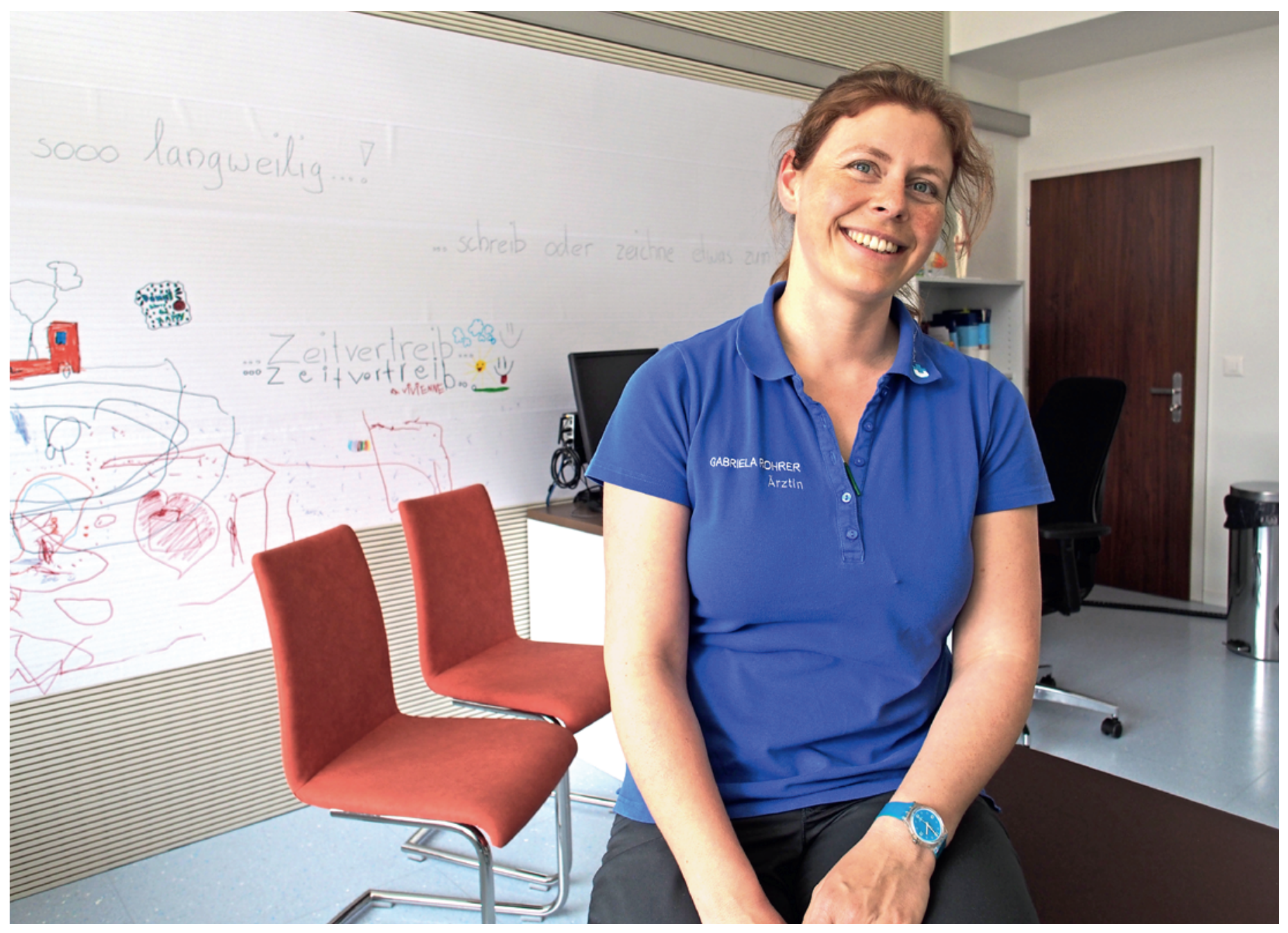


ein, und wir werden uns die Praxisapotheke genau ansehen. Sicher werde ich versuchen, Antibiotika etwas zurückhaltender einzusetzen als mein Vorgänger», sagt Gabriela Rohrer. Besonders wichtig ist ihr das Thema Abgrenzung: "Paul war praktisch immer während 24 Stunden verfügbar», erzählt sie, "viele hatten seine Handynummer und riefen ihn Tag und Nacht an. Und wer telefonierte, erhielt in der Regel auch gleich einen Termin.» Das wolle sie restriktiver handhaben, betont Gabriela Rohrer, nicht nur wegen der oft langen Wartezeiten, die so für die Patienten manchmal entstanden seien. «Es geht auch um meine ganz persönliche Energiebilanz. Wenn ich gut zu meinen Patientinnen und Patienten schauen will, muss ich auch gut zu mir selber schauen.»

Eine Feststellung, die die junge Frau mit grossen, offenen Augen, direktem Blick und ruhiger, beinahe dozierender Stimme auf den Tisch legt und sie fast ein wenig altklug wirken lässt. Eine Erkenntnis vor allem aber, die sie in ihrer noch kurzen Karriere schon selber gewonnen, am eigenen Leib erfahren hat. "Auf sich selber zu hören, lernt man im Medizinstudium nicht», sagt Gabriela Rohrer. Grosses Bedauern und etwas Trotz schwingen mit.

\section{Prägende Erinnerungen}

Rohrer hatte auf einer Farm in Kanada gearbeitet und in der Schweiz ein Bauernlehrjahr absolviert, bevor sie Medizin zu studieren begann, und sie war als Handlangerin auf dem Bau tätig gewesen. Forstwirtschaft oder Agronomie waren mögliche Studienrichtungen. «Mein erstes Studienjahr in der Medizin war also so oder so ein Kulturclash", kommentiert sie. "Alles war sehr abstrakt, hatte wenig Bezug zum Alltag.» Auswendiglernen sei nicht ihre Sache gewesen, sie habe - in der

\section{Endlich eine neue Hoffnung für die medizini- sche Grundversorgung in dieser Gegend.}

ursprünglichen Bedeutung des Wortes - das Begreifbare gesucht. Schliesslich sei es ja auch das Erlebnis einer «Metzgete» auf einem Bauernhof gewesen, das in ihr die Faszination für die Medizin geweckt habe. Ein Schwein wurde geschlachtet.

«Das Gewebe, die Organe, die da auf dem Tisch lagen, animierten mich zur Frage, wie aus einer Zelle ein Organismus wird, wie Leben entsteht, wie das alles funktioniert.» Und sie fügt, wiederum mit einem herzhaften Lachen, bei: «Ich war immer ein neugieriges Kind. Aber auch ein sensibles.»

Ihre Erlebnisse als Studentin mit Arbeiten im Seziersaal kommen ihr in den Sinn, auch hier sei sie faszi-

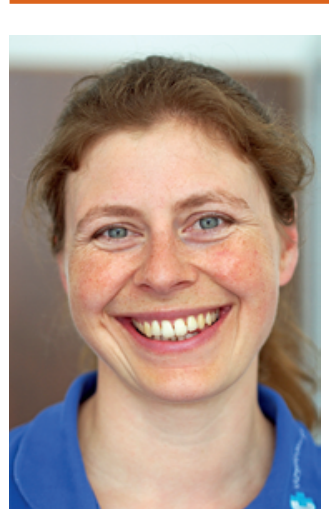

\section{Zur Person}

Dr. med. Gabriela Rohrer wurde 1983 in Bern geboren. Sie wuchs in Stettlen auf und absolvierte nach der Matura ein Zwischenjahr. Einige Monate arbeitete sie auf einer Farm in Kanada und temporär auf dem Bau. In Utzigen absolvierte sie ein Bauernlehrjahr. 2003 bis 2009 studierte sie Medizin in Bern. Nach ihrem Abschluss war sie am Spital Aarberg, am Kantonsspital St. Gallen und am Spital Wil tätig sowie in Hausarztpraxen in Lauterbrunnen, Degersheim und Wildhaus. Seit 2016 ist sie Präsidentin des Vereins «Junge Hausärztinnen und -ärzte Schweiz». Im Sommer 2017 stiess sie zum Team der Hausarztpraxis in Flühli Sörenberg, am 1. Januar dieses Jahres übernahm sie die Praxis mit insgesamt 12 Angestellten von ihrem Vorgänger Paul Affentranger, der sie rund 40 Jahre lang geführt hatte. Gabriela Rohrer ist mit Pablo Siegenthaler liiert, der für die Administration der Praxis verantwortlich ist.

niert gewesen, "wie in einer anderen Welt». Bloss: «Nachher hatte ich regelmässig Albträume, aber die interessierten niemanden.» Wir hätten doch eine natürliche Abscheu, wenn es darum gehe, Leichen zu berühren, erklärt sie. «Leichen zu zerstückeln, empfinden wir als eine Schändung, als etwas Perverses. Und pervers ist es auch, so zu tun, als würde das in uns nichts auslösen.»

Zum Thema «Betroffenheit» taucht ein zweites Erlebnis aus der Studienzeit auf. Einer Patientin habe sie eine Krebsdiagnose verkünden müssen. «Das macht mir Mühe», habe sie dem zuständigen Assistenzarzt anvertraut. Seine Antwort: «Wenn du mit dem nicht umgehen kannst, bist du hier am falschen Ort.»

\section{Der Blick auf sich selber}

Gabriela Rohrer versuchte stets, auf ihre innere Stimme zu hören. Zu sich selber zu schauen, gelang ihr nicht immer. An die Weiterbildung in der Chirurgie eines kleinen Spitals hat sie schlechte Erinnerungen: «Stunden zu bolzen gehörte zum guten Ton und führte $\mathrm{zu}$ einem enormen Gruppendruck. Schnell, schnell eine Pizza auf dem Spitaldach gehörte zum höchsten der Gefühle.» Aber das Motto laute: Vogel friss oder stirb, «deshalb lassen sich viele Ärzte auch von solchen Arbeitsbedingungen gängeln». Als sie Assistenzärztin in der Inneren Medizin eines Zentrumsspitals war, fiel sie in eine Erschöpfungsdepression - und drei Monate lang aus. «Verschiedene ungünstige Faktoren kamen zusammen», analysiert sie heute. "Zum Beispiel der Umzug in eine mir fremde Gegend und das Gefühl, im 
Spital eine Nummer zu sein. Auch die Patienten waren Nummern. Man sprach nicht mit ihnen - oder höchstens so, dass sie nichts verstanden.» Zwei markante und aus ihrer Sicht typische Sätze aus dieser Zeit hat sie nicht vergessen: «Der Kardiologe tut, was der Kardiologe gelernt hat.» Was für alle Disziplinen gelte und doch eine wohl etwas gar enge Sichtweise sei. Und: «Dieser Patient ist eine Leihgabe der Intensivstation.» Trotz allem blieb sie der Medizin treu. «Nach meinem Praktikum am Ende des Studiums wusste ich: Ich werde Hausärztin, und zwar auf dem Land.» Das Strahlen auf ihrem Gesicht unterstreicht, dass es die richtige Entscheidung war.

Die Frage nach ihrem Selbstverständnis und ihren verschiedenen Rollen als Ärztin machte sie zum Thema ihrer Doktorarbeit. «In welcher Beziehung stehe ich zum Patienten? Was bedeutet dies für mich als Partnerin, Kollegin, Frau? Wie steht es mit der Selbstaufopferung? Der Fehlerkultur? Und dem Umgang mit meinen eigenen Emotionen?» Solche Fragen versuchte sie zu beantworten. Und solche Fragen stellt sie sich nach wie vor regelmässig.

«Empathie ist für einen Arzt wie ein ganz wertvolles Hochpräzisionsinstrument. Etwas so Wichtiges kann ich doch nicht einfach in eine Ecke stellen und vergammeln lassen, ich muss es hegen und pflegen, und deshalb muss ich auch immer wieder über meine eigene Befindlichkeit, meine Unzulänglichkeiten und Ängste offen sprechen", konstatiert Gabriela Rohrer - und ist einmal mehr beeindruckend klar und erstaunlich weise. «Immer wieder begegne ich eigenen Unzulänglichkeiten, immer wieder besteht die Gefahr, etwas zu übersehen oder zu verpassen.» Wenn sie ihre bisherigen Erkenntnisse als junge Medizinerin in einem Fazit zusammenfassen müsste, sagt sie, würde sie sagen: «Es ist schwierig, ein guter Doktor zu sein. Aber es macht mir Spass, Hausärztin zu sein.»

\section{Interessantes Finanzierungsmodell}

Im Moment stehen philosophische Fragen in dieser Praxis allerdings nicht zuoberst auf der Prioritätenliste. Es geht in erster Linie um Handfestes. «Wollen wir diese Gipsbänder noch behalten?», fragt eine der Praxisassistentinnen. Gabriela Rohrer und ihr Partner, der sich um die Administration kümmert, fragen sich beispielsweise: Wie werden wir finanziell über die Runden kommen? Speziell in diesem Fall: Das ganze Dorf, die ganze Gegend machte sich zu diesem Thema Gedanken, als klar wurde, wie schwierig eine Nachfolgeregelung für die lokale Arztpraxis ist - man sammelte Geld und gründete eine Genossenschaft. Gemeinde und Kanton, aber auch Private engagierten sich. Sie kauften zwei Stockwerke des Praxisgebäudes und finanzierten den dringend nötigen Umbau. Die junge Ärztin gründete zusammen mit ihrem Lebenspartner eine Aktiengesellschaft, die nun Räumlichkeiten und Infrastruktur bei der Genossenschaft mietet. Dies erspart die hohen Investitionskosten, die für einen Kauf der Praxis nötig wären. Trotzdem braucht es laut Rohrer ein Startkapital von rund 300000 Franken, um den

«Es geht auch um meine ganz persönliche Energiebilanz.»

laufenden Betrieb aufrechtzuerhalten. Ein Kontokorrent bei der örtlichen Bank deckte einen Grossteil dieses Betrages ab. Das Einlagekapital für die AG-Gründung konnte das junge Paar aus den eigenen Ersparnissen und privaten Darlehen zusammenlegen. «Finanziell bin ich also nicht abhängig und trage kaum ein Risiko», bilanziert Gabriela Rohrer zufrieden.

\section{... und ein Kind?}

Andere Belastungen wiegen schwerer, und auch darüber spricht diese junge Frau in aller Offenheit. «Eigentlich wollten wir mit einem Kind hierher kommen», erzählt sie. Es hat - noch - nicht sein sollen, sie verlor ihr Kind während der Schwangerschaft. Mutter zu werden sei als Projekt jedoch keineswegs gestorben, ergänzt sie, jetzt sei einfach nicht gerade der richtige Moment dafür. «Es stimmt nicht, dass man immer für alles Zeit hat. Es gibt Phasen im Leben. Für uns ist ein Baby im Moment ein bewusster Verzicht - während eines begrenzten Zeitraums.» So, wie zurzeit auch das Wandern oder der Tanz zu kurz kämen. «Das muss sich in absehbarer Zeit aber ganz klar wieder ändern", sagt sie bestimmt.

«Das Dümmste, was mir jetzt passieren könnte, wäre, dass ich selber krank und arbeitsunfähig würde.» Ein gewisser Druck ist also trotz allem da, «und die Erwartungen rundherum sind hoch. Das macht bisweilen auch Angst. Aber ohne Angst kein Mut.» Übrigens: Kraft zu ihrem Schritt habe sie wesentlich in ihrer «Peergroup» erhalten, dem Verein «Junge Hausärztinnen und -ärzte Schweiz», den sie seit 2016 präsidiert. «Da gibt es Leute, die mich immer wieder trugen und für mich nach wie vor eine Heimat sind.»

Und: Kraft hätten ihr ihre künftigen Patientinnen und Patienten gegeben: «Mir ist sehr viel Goodwill entgegengekommen. Dass ich hier willkommen bin, ist gerade für mich speziell, gehöre ich doch der «NoFuture-Generation` an. Schon in der Sekundarschule versuchte man mir klarzumachen, dass in der Berufswelt niemand auf mich wartet.» Und ob! 